\title{
Contingency management for smoking cessation in pregnancy
}

Jacob L. Miller MD ${ }^{1}$, Brady Reynolds $\mathrm{PhD}^{2}$, Courtney Crain MD ${ }^{1}$, Hisham Keblawi MD, FACOG $^{1}$, Lynne J. Goebel MD ${ }^{1}$

\section{Author Affiliations:}

1. Marshall University Joan C. Edwards School of Medicine, Huntington, West Virginia

2. University of Kentucky, Lexington, Kentucky

The authors have no financial disclosures to declare and no conflicts of interest to report.

\section{Corresponding Author:}

Jacob L. Miller, MD

Department of Surgery

Marshall University Joan C. Edwards School of Medicine

Huntington, West Virginia

Email: millerja@marshall.edu 


\begin{abstract}
This pilot study examined the feasibility of applying Contingency Management (CM), where the woman receives financial compensation for abstinence from smoking. Eleven subjects were recruited from Marshall's OB clinic for the program. CO levels were measured daily using online video recording. Quit rates were determined at the end of pregnancy. Birth outcomes were measured at delivery. 21 pregnant smokers from Marshall's OB clinic were used as the control group. The results demonstrated a trend toward improved rates of smoking abstinence in CM participants as compared with the control group. Our study also showed a trend towards improved birth outcomes in the CM group. However, the results were not statistically significant, and we believe a larger study in our population is warranted.
\end{abstract}

\title{
Keywords
}

smoking, pregnancy, Appalachia, contingency management, birth outcomes

\section{Introduction}

We have evidence of the harmful effects on the fetus of maternal smoking during pregnancy from as early as 1957, when maternal smoking was found to be associated with prematurity. ${ }^{1}$ Although smoking during pregnancy has decreased in recent decades, it is still considered the top preventable cause of poor birth outcomes in the United States. ${ }^{2,3}$ Smokers have an increased risk of ectopic pregnancy, spontaneous abortion, placental abruption, placenta previa, preterm birth, low birthweight (LBW), stillbirth, and sudden infant death syndrome (SIDS). After birth, the children of smokers are at increased risk for behavioral problems, attention deficit disorder, hyperactivity, learning disability, obesity, elevated blood pressure, diabetes, and an increased risk of becoming smokers themselves. ${ }^{3}$ Additionally, the newborn is at increased risk for cognitive problems, ear infections, bronchitis, pneumonia, asthma, and SIDS if exposed to secondhand smoke postpartum. ${ }^{4}$

Although the rate of maternal tobacco use during pregnancy has dropped, certain populations such as Appalachians still have high rates of maternal tobacco use during pregnancy and associated high rates of poor birth outcomes. For example, in a study of more than 4,100 women surveyed in Appalachia, 25\% were self-reported as smokers compared to the national rate of $12 \% .^{5}$ Additionally, babies born to rural women were on average 700 grams lighter and were born more than three weeks earlier. Unfortunately, rural women are less likely to use prenatal care; this is due to many factors, one of which is increased travel time to medical provider. ${ }^{5}$

Contingency Management (CM), a program where the woman receives financial compensation as a reward for abstaining from smoking, has proven particularly effective in reducing smoking during pregnancy with up to $40 \%$ of participants achieving 
abstinence by late pregnancy. ${ }^{2}$ The web-based version of this program is ideal in the rural population because it lessens the travel burden and provides frequent verification and reinforcement, two essential components for smoking abstinence that promote rapid change in behavior. ${ }^{6}$

The primary aim of the study was to determine the benefit of $\mathrm{CM}$ on maternal smoking abstinence compared to pregnant smokers not enrolled in this program. Based on what we know about pregnant smokers, we hypothesize that the group receiving CM will achieve higher abstinence rates at delivery than pregnant smokers not enrolled in CM. Furthermore, this study assessed secondary outcomes such as birth weight, gestational age at birth, and NICU/NTU admissions.

\section{Methods}

In this pilot study, we recruited eleven pregnant smokers from the Marshall Health Obstetrics Clinic, located in Huntington West Virginia, a state entirely located in Appalachia. Participants were between the ages of 18 and 35 in their first 15 weeks, did not use recreational drugs aside from marijuana and had no history of diabetes. Marshall University IRB approved this study.

Smoking status was defined as smoking at least two cigarettes per day and was confirmed via urine cotinine (>200ng/mL) or exhaled CO values (>10 parts per million (ppm)) at initial clinic visit. Recreational drug use was assessed using a urine test. Gestational age was determined using the date of last menstrual period (15 weeks or less) if ultrasound was not available.

Marshall's OB clinic was the source of the control group as it is the clinic from which the members of the CM group were recruited. We reviewed the charts of all 210 labor and delivery patients seen during the same time frame as the CM group. Of those charts, 21 patients met the screening criteria for the control group. Patients included were women aged 18-35 in their first 15 weeks who were self-reported smokers, denied recreational drug use aside from marijuana, had no history of diabetes, and would deliver in approximately the same time frame as the women in the active study group. Patients were excluded for incarceration, loss to follow up, and abortion.

Using a provided computer with internet access, subjects participated in a contingency management $(\mathrm{CM})$ program wherein they received financial compensation for reductions in cigarette use. Participants were required to videotape themselves twice daily giving a breath sample to determine breath $\mathrm{CO}$ using a provided monitor and to submit this videotape electronically to the investigators for review. Participants uploaded videos to the Motiv8 system using unique logins and passwords. Video submissions took 1-2 minutes to record and had an 8-hour inter-sample interval. To leave a $\mathrm{CO}$ sample, participants showed the $\mathrm{CO}$ meter to the web camera, initiated the $\mathrm{CO}$ countdown screen, and held their breath for 15 seconds. They then exhaled into the meter, and displayed the meter to the camera. Patients received monetary compensation based on the contingency management protocol listed in Figure 1. 
Figure 1. Contingency management timeline

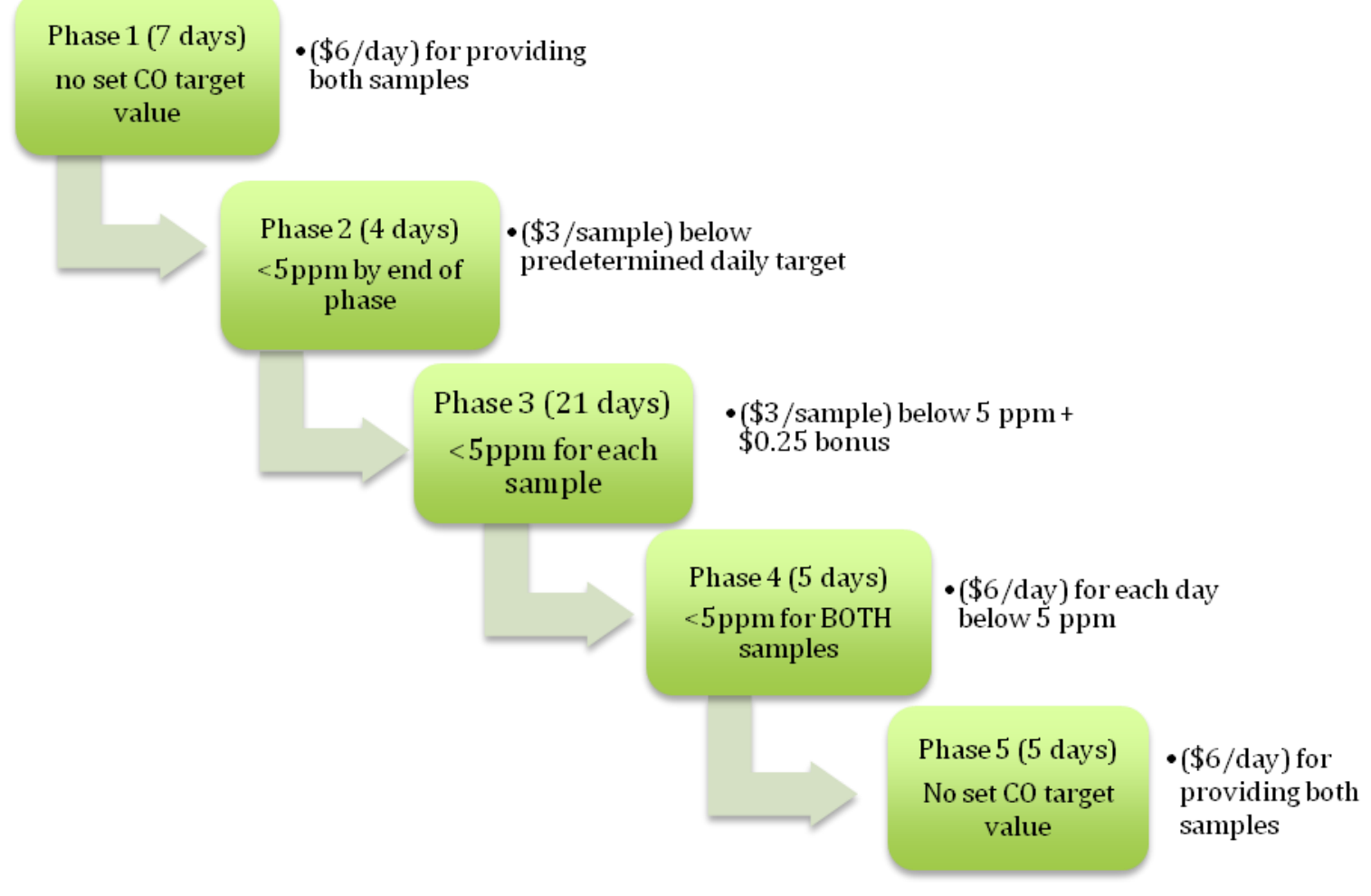

During phase 1 of the CM program, which lasted 7 days, women received $\$ 6$ per day for providing two $\mathrm{CO}$ recordings on time regardless of $\mathrm{CO}$ value. These $\mathrm{CO}$ values were used to determine daily target values for the next phase, with a required reading below 5 ppm as the final target value. Phase 2, which lasted 4 days, required participants to provide two CO breath samples per day, and participants were provided $\$ 3$ for each breath sample with $\mathrm{CO}$ values below these predetermined target values. During phase 3 , participants were provided $\$ 3$ for each CO breath sample below $5 \mathrm{ppm}$. Additionally, rewards were escalated by $\$ 0.25$ for each consecutive breath sample below 5 ppm during this time. If a $\mathrm{CO}$ value was $5 \mathrm{ppm}$ or more during this time, the reward amount was reset to $\$ 3$ for the next $\mathrm{CO}$ value below $5 \mathrm{ppm}$. Phase 3 lasted 21 days. Phase 4 provided $\$ 6$ per day for participants who provided two CO breath samples. Phase 4 lasted 5 days. Phase 5 provided $\$ 6$ per day for providing both $\mathrm{CO}$ breath samples on time, regardless of $\mathrm{CO}$ value. Phase 5 lasted 5 days.

The outcome data at birth including gestational-age at birth, birth weight, APGAR scores, and maternal smoking status were collected from the medical record. In addition, infant ICU visits in the first six months were documented. At monthly prenatal visits and the 6 week postpartum follow-up visit, urine cotinine, exhaled $\mathrm{CO}$, smoking rate over the previous two weeks, ratings of nicotine withdrawal, and smoking urges were assessed in the CM group. Patients were compensated 10 dollars for each visit.

Our results were analyzed using Microsoft Excel. T-tests were calculated using Vassarstats.net on the "t-test for independent samples" section. Categorical data was analyzed using Fisher's exact. 


\section{Results}

The average age of our pregnant smokers was 26 in the CM group and 25 in the control group. T-test (0.69) showed no statistically significant difference in these values. There were 10 patients that self-identified as "white" and one participant that identified as "other" in the CM group. Racial data was not collected in the control group.

Table 1. Participant Substance Use

\begin{tabular}{|l|c|c|c|}
\hline Substance & $\begin{array}{c}\text { CM \%(n) } \\
(\mathrm{n}=11)\end{array}$ & $\begin{array}{c}\text { Control \%(n) } \\
(\mathrm{n}=21)\end{array}$ & P Value \\
\hline marijuana & $9[1]$ & $10[2]$ & $\mathrm{NS}$ \\
\hline buprenorphine/naloxone & $18[2]$ & $25[5]$ & $\mathrm{NS}$ \\
\hline methadone & $9[1]$ & $10[2]$ & $\mathrm{NS}$ \\
\hline opiates & $0[0]$ & $5[1]$ & $\mathrm{NS}$ \\
\hline oxycodone & $0[0]$ & $5[1]$ & $\mathrm{NS}$ \\
\hline
\end{tabular}

Table 1 shows participant and control substance use from urine dipsticks of samples at delivery. Both groups had marijuana use and buprenorphine or methadone use; however, only the control group had a participant positive for opiates and oxycodone. Fisher exact performed to attain p-value.

* Urine drug screen was not performed in one patient in the control group and was excluded from results in this section.

Table 2. CM Participant Outcomes

\begin{tabular}{|l|l|l|l|l|}
\hline & $\begin{array}{l}\text { Enrollment } \\
\text { Status }\end{array}$ & $\begin{array}{l}\text { Participation (Percent of } \\
\text { required CO recordings } \\
\text { submitted) }\end{array}$ & $\begin{array}{l}\text { Compensation } \\
\text { (In Dollars) }\end{array}$ & Quit \\
\hline MUOB1 & Withdrew & 6 & 22.00 & Yes \\
\hline MUOB2 & Withdrew & 17 & 49.00 & No \\
\hline MUOB3 & Completed & 86 & 234.50 & Yes \\
\hline MUOB4 & Completed & 32 & 146.50 & No \\
\hline MUOB5 & Completed & 81 & 126.75 & No \\
\hline MUOB6 & Completed & 49 & 132.00 & No \\
\hline MUOB7 & Completed & 100 & 259.75 & Yes \\
\hline MUOB8 & Completed & 100 & 418.50 & Yes \\
\hline MUOB9 & Completed & 22 & 49.00 & No \\
\hline MUOB10 & Completed & 98 & 237.75 & Yes \\
\hline MUOB11 & Withdrew & 11 & 22.00 & No \\
\hline
\end{tabular}

Table 2 shows the results for the CM group. Three of the 11 patients withdrew from the study, but smoking status at delivery was retrieved from their medical records. Only 2 participants submitted all of the requested $\mathrm{CO}$ readings. Four of the 5 patients with over $80 \%$ compliance with the program were able to quit smoking. 
Table 3. Maternal Cessation Rates and Birth Outcomes

\begin{tabular}{|l|c|c|c|}
\hline Birth Characteristics & $\begin{array}{c}\text { CM group } \\
(\mathrm{n}=11)\end{array}$ & $\begin{array}{c}\text { Control } \\
(\mathrm{n}=21)\end{array}$ & P Value \\
\hline Maternal abstinence rate at delivery \%(n) & $45(5)$ & $19(4)$ & 0.21 \\
\hline Average birth weight $(\mathrm{g})$ & 3466 & 3166 & 0.10 \\
\hline Average gestational age (weeks) & 39.2 & 38.9 & 0.63 \\
\hline Average number of ICU visits & 0.19 & 0.57 & 0.13 \\
\hline Average APGAR & 8.72 & 8.1 & 0.22 \\
\hline
\end{tabular}

Table 3 shows birth outcomes. There was a trend towards higher average birth weight and average gestational age, and fewer NICU visits in the CM group compared to controls as shown in table 3 . Also, $45 \%$ of the CM group was abstinent at delivery compared to $19 \%$ of controls.

Figure 2: CM adherence among those who completed program

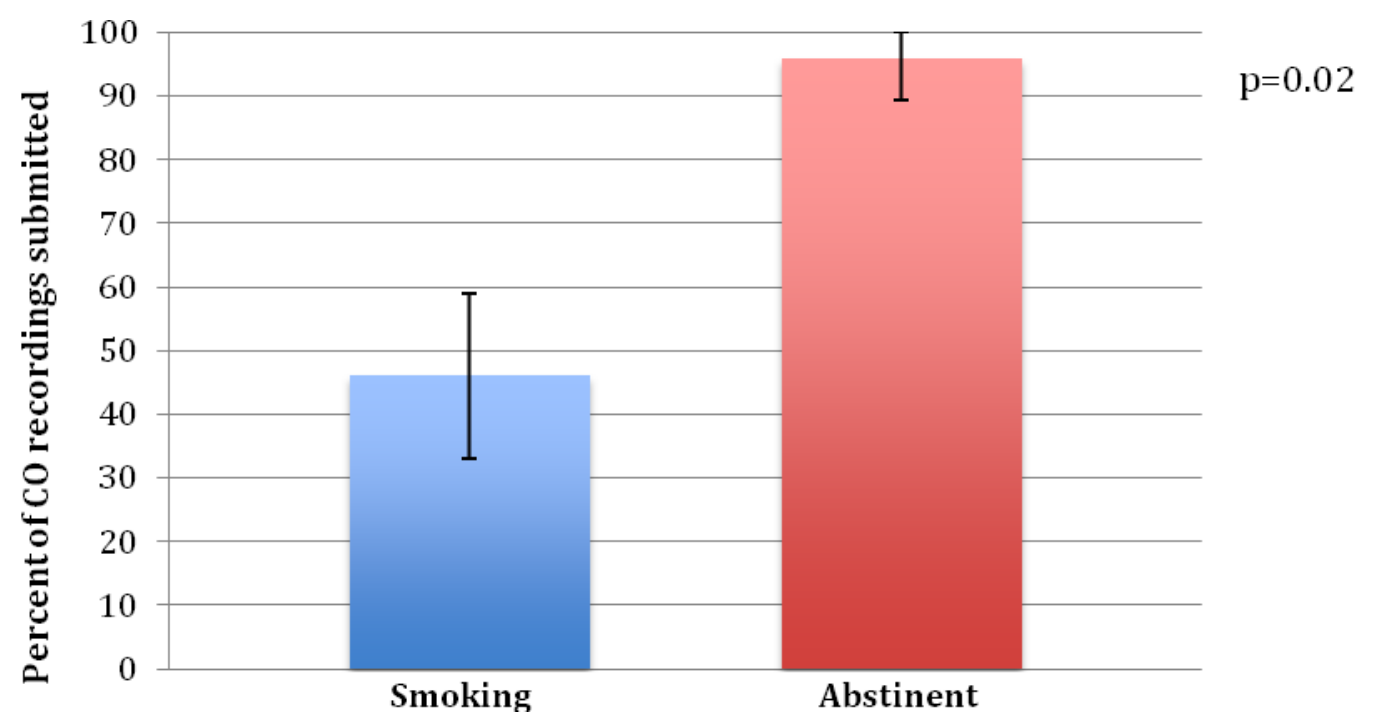

Figure 2 shows program adherence rates for quit vs smoking at delivery among those who completed CM program. Adherence was greater in those who were able to quit smoking compared to those who did not quit $(\mathrm{p}=0.02)$. Overall adherence was $55 \%$. Among those who completed the program, adherence was $71 \%$.

\section{Discussion:}

Smoking during pregnancy is considered the top preventable cause of poor birth outcomes in the United States. However, smoking cessation resources are often difficult for the rural pregnant smoker to access due to economic and geographic obstacles. Previous studies have examined the feasibility of web-based CM in rural pregnant smokers, with positive results. In this pilot study in our Appalachian population, we showed a trend towards benefit of a home-based contingency management program for pregnant smokers on improving maternal smoking abstinence and birth outcomes as 
compared to pregnant smokers not enrolled in this program. The benefit of smoking cessation was statistically significant in those who most adhered to the program.

Our population had high rates of buprenorphine/methadone use and opiate drug abuse as demonstrated by urine drug testing at delivery in both the control and CM groups. This altered the birth outcome data as neonatal abstinence syndrome (NAS) mandates a NICU/NTU visit. In all cases, the reason listed in the medical records for NICU/NTU visits was for NAS. In addition, NAS and maternal tobacco use are both associated with low-birth weight (LBW). Only one infant in the study was classified as LBW $(<2500 \mathrm{~g})$ and this was in the control group. Still, it is difficult to draw conclusions about the contributing factors to this outcome given the small scale of the study $(n=33)$. In a previous study, cigarette smoking in mothers taking opiate agonists was associated with longer NICU stays and increased morphine requirements to treat NAS. ${ }^{7}$ In our study, it is difficult to determine whether complications from fetal exposure to maternal tobacco use may have led to additional NICU/NTU admissions since our study was not designed to detect this difference. Treatment for NAS may last as long as a month in some cases, possibly preventing detection of additional ICU visits due to complications from maternal tobacco smoking.

Investigators struggled with recruitment and retention of participants. The most commonly cited reasons for withdrawal from the study were stress and inability to commit time required to participate. Adherence to the CM program was lower in our program $(55 \%)$ when compared to other programs which may be a reflection of our high risk population. For example, previous studies have shown a $65 \%$ adherence in adult rural smokers in $\mathrm{CM}$ programs with similar $\mathrm{CO}$ monitoring requirements. ${ }^{8}$ Among those who completed the program, we observed higher quit rates in those with greater adherence, which may indicate the importance of adherence in successful abstinence. Some studies that had more intensive CO monitoring requirements showed a lower $(46 \%)$ adherence indicating more intensive monitoring could be a barrier to success. ${ }^{8}$ No instances of withdrawal from the program were attributed to inaccessibility or lack of needed resources (internet access, electricity, equipment, etc.), which is similar to other programs. However, our use of a laptop computer may not have been convenient for the patient, especially if they were away from home. Future research could test a contingency management app that could be used on a smart phone to see if this increases compliance with the program.

One goal of our pilot study was to determine the benefit of CM in our population. We believe we have achieved this goal and a larger study in our population is warranted. Overall, we showed a trend towards improved rates of smoking abstinence during pregnancy in the CM group (0.45) as compared to routine care (0.19). However the scale of this pilot study was small, and there was not enough power to demonstrate a statistically significant association between CM and smoking abstinence during pregnancy. Previous studies have shown high rates (up to 40\%) of smoking abstinence in late pregnancy for patients enrolled in CM. Our quit rate (45\%) is similar. ${ }^{8}$ Our study also showed a trend in greater gestational age at birth, birth weights, APGAR scores, and fewer ICU visits in the CM group, which may be associated with the group's greater rate 
of smoking abstinence. These results were not statistically significant, which may be the result of the small size of our study. Some problems encountered with implementation of this program in our population include poor retention, low adherence, and a high prevalence of opiate drug use that may have skewed our data. Some solutions for these issues include adopting more accessible modalities for monitoring smoking abstinence (i.e. smart phone apps) to improve compliance and adherence as well as increasing the size of future studies to more effectively investigate the effects of CM on maternal cessation rates and birth outcomes. 


\section{References}

1. Simpson WJ. A preliminary report on cigarette smoking and the incidence of prematurity. Obstetrical \& Gynecological Survey. Am J Obstet Gynecol. 1957;12(6):868-9.

2. Higgins ST, Washio Y, Lopez AA, Heil SH, Solomon LJ, Lynch ME, et al. Examining two different schedules of financial incentives for smoking cessation among pregnant women. Prev Med. 2014;68: 51-7.

3. Rogers JM. Tobacco and pregnancy: Overview of exposures and effects. Birth Defects Res C Embryo Today: 2008;84(1):1-15.

4. Cnattingus S. The epidemiology of smoking during pregnancy: Smoking prevalence maternal characteristics, and pregnancy outcomes. Nicotine Tob Res. 2004;6:125-40.

5. Bailey BA, Cole LKJ. Rurality and Birth Outcomes: Findings From Southern Appalachia and the Potential Role of Pregnancy Smoking. J Rural Health. 2009;25(2):141-9.

6. Harris M, Reynolds B. A pilot study of home-based smoking cessation programs for rural, Appalachian, pregnant smokers. J Obstet Gynecol Neonatal Nurs. 2015;44:236-245.

7. Jones H, Heil SH, Tuten M, Chisolm MS, Foster JM, O'Grady KE, Kaltenbach K. Cigarette smoking in opioid-dependent women: Neonatal and maternal outcomes. Drug Alcohol Depend. 2013;131:271-277.

8. Reynolds B, Harris M, Sloan SA, Shelton BJ, Dallery J. A feasibility study of home-based contingency management with adolescent smokers of rural Appalachia. Exp Clin Psychopharmacol. 2015;23(6): 486-493. 